\title{
The atomistic structure and energy of nascent dislocation loops
}

\author{
V B Shenoy $\dagger$, M Ortiz $\ddagger$ and R Phillips $\dagger$ \\ † Division of Engineering, Brown University, Providence, RI 02912, USA \\ \$ Graduate Aeronautical Laboratories, Caltech, Pasadena, CA 91125, USA
}

Received 21 September 1998, accepted for publication 26 May 1999

\begin{abstract}
An harmonic lattice theory is used, in conjunction with Mura's theory of eigendistorsions, to study the structure and energetics of nascent dislocation loops in face-centredcubic (FCC) crystals. An analytical expression for the activation energies of such loops is derived. The results obtained herein indicate that thermal activation of small dislocation loops is possible at high stress levels such as those found in the vicinity of a crack tip. The implications of these results in understanding phenomena such as the brittle-ductile transition are discussed.
\end{abstract}

\section{Introduction}

The role of small dislocation loops in determining the plastic behaviour of crystalline solids has recently been of renewed interest. The thermally-assisted nucleation of large numbers of small dislocation loops has long been thought to underly the melting of crystals (Nabarro 1967). Recently, Khantha et al (1994) proposed that the cooperative instability of a collection of large numbers of dislocation loops causes the brittle-ductile transition (BDT) in crystals. They argue that the presence of a large number of loops in the solid reduces the effective shear modulus of the solid, and this makes the formation of new loops energetically cheaper. The fall in the modulus is, they observe, larger at higher temperatures due to the presence of a larger population of thermally activated dislocation loops. At some critical temperature this process becomes self-sustaining and the collective expansion of these loops occurs in an unstable manner. In a two-dimensional (2D) solid free of external stress this corresponds to a Kosterlitz-Thouless-type instability (Kosterlitz and Thouless 1973). The analysis given by Khantha et al (1994) considers a 2D solid under an external stress, and predicts a critical temperature well below the melting temperature of the solid. For example, in silicon, Khantha et al (1994) predicted the critical temperature to be about $900 \mathrm{~K}$ at stress levels of a tenth of the elastic modulus, which is in excellent agreement with the experimentally measured value of the BDT temperature of silicon. They, therefore, conclude, on the basis of their 2D analysis, that the BDT is a manifestation of a stress-assisted cooperative instability in the population of dislocation loops.

Crucial in the argument presented by Khantha et al (1994) is the presence of a larger number of thermally activated dislocation loops at temperatures below those which result in a significant reduction of the elastic moduli of the crystal. In three dimensions this requires that the activation energy of a dislocation loop is of the order of $k T$, where $k$ is Boltzmann's constant and $T$ is the absolute temperature. Kroupa (1966), for example, has given the energy of a slip loop of radius of an isotropic solid as

$$
\varepsilon=\frac{\mu(2-v)}{4(1-v)} b^{2} r\left(\log \frac{4 r}{r_{0}}-2\right)
$$


where $\mu$ is the shear modulus, $v$ is Poisson's ratio, $b$ is the Burgers vector and $r_{0} \sim b$ is the core cut-off. it is important to note that this equation is useful only in the estimation of the elastic energy of large loops, $r \gg r_{0}$. The energy estimates derived from Kroupa's equation are of the order of $\approx 10-100 \mathrm{eV}$, which would certainly rule out thermal activation for loops of sizes, say, $r>10 b$. However, linear elasticity, and by extension Kroupa's formula, is inapplicable to small loops of size comparable to the lattice parameter. Indeed, quantities such as the radius of the loop are themselves ill-defined in that limit. Consequently, attempts to assess the plausibility of the Khantha et al (1994) theory based on linear elasticity are a fortiori inconclusive.

The singularities that cripple continuum analyses are naturally absent in discrete lattice theories (see, e.g., Born and Huang 1954). In particular, formulations based on the harmonic approximation are analytically tractable by Fourier transform methods. While harmonic theories have proven successful in treating the dynamical properties of perfect crystals, they at first appear unsuitable for studying lattice defects in consequence of the quadratic and convex character of the total energy of the lattice when regarded as a function of the displacements of the atoms. Indeed, the convexity of the energy function in effect precludes the spontaneous nucleation of defects in the lattice. This limitation of harmonic lattice statics may be overcome by the eigendistorsion method of Mura (1987). In this method, defects such as dislocations are represented as incompatible distortions, or eigendistorsions, of the lattice. Such eigendistorsions, when uniform, define lattice preserving deformations of the crystal and are, therefore, energy minimizers. The incompatibility of the eigendistorsions corresponding to defects, however, inevitably induces a state of residual deformation in the lattice. The energy attendant to that residual deformation is the energy of the defect.

By taking full account of the discreteness of the lattice, the theory of eigendistorsions provides an analytically tractable means of analysing nascent dislocation loops in crystals and estimating their energies which is free of the limitations of linear elasticity. In this paper we apply the theory of eigendistorsions to the study of nascent dislocation loops in face-centredcubic (FCC) crystals. The activation barrier for the formation of a small dislocation loop is obtained analytically. Our principal finding is that the nucleation of small loops may indeed occur by thermal activation under the action of sufficiently large stresses, for example, such as arise in the vicinity of crack tips.

The paper is organized as follows. Section 2 lays down the notation and records the mathematical tools required for subsequent analyses. Section 3 introduces the concept of eigendistorsion and develops a theory for the formation of dislocation loops in crystals. Section 4 contains a discussion of a screw dislocation dipole in a square lattice and validates the continuum theory for the same. The main application of the theory presented in section 5 concerns the study of nascent loops in FCC crystals. Finally we summarize our findings and conclude the paper in section 6 .

\section{Lattice statics prolegomena}

\subsection{Simple Bravais lattices}

A simple Bravais lattice is a set of points in space

$$
x(l)=l^{i} a_{i}
$$

where $\boldsymbol{l} \in Z^{3}$ is a multiindex and $\left(\boldsymbol{a}_{1}, \boldsymbol{a}_{2}, \boldsymbol{a}_{3}\right)$ is some suitable lattice basis. The dual basis $\left(\boldsymbol{a}^{1}\right.$, $\left.\boldsymbol{a}^{2}, \boldsymbol{a}^{3}\right)$ and the reciprocal basis $\left(\boldsymbol{b}^{1}, \boldsymbol{b}^{2}, \boldsymbol{b}^{3}\right)$ may be obtained using standard definitions such that $b^{i}=2 \pi a^{i}$. The volume of the unit cell of the lattice is assumed to be $V$. Consequently, the 
unit cells of the dual and reciprocal bases have volumes $1 / V$ and $(2 \pi)^{3} / V$, respectively. There is no unique choice for a lattice basis, for another triad $\left(\boldsymbol{a}_{1}^{\prime}, \boldsymbol{a}_{2}^{\prime}, \boldsymbol{a}_{3}^{\prime}\right)$ obtained by application of a linear transformation of the original basis such as

$$
\boldsymbol{a}_{i}^{\prime}=A_{i}^{j} \boldsymbol{a}_{j}
$$

also defines a lattice basis, with the proviso that $A_{i}^{j} \in Z$ and

$$
\operatorname{det} \boldsymbol{A}= \pm 1 \text {. }
$$

These transformations also define crystal preserving homogeneous deformations of the lattice and will prove to be important in the applications that follow. Thus, affine mappings of the form

$$
\boldsymbol{y}=\boldsymbol{F} \boldsymbol{x}
$$

such that

$$
\boldsymbol{F}^{\mathrm{T}}=A_{i}^{j} \boldsymbol{a}^{i} \otimes \boldsymbol{a}_{j}
$$

map lattice points into lattice points. Integer matrices which are rank-one connected to the identity, i.e. matrices of the form

$$
A_{i}^{j}=\delta_{i}^{j}+m_{i} l^{j}
$$

with $m, l \in Z^{3}$, are of particular significance to dislocations. Proviso (3) is satisfied provided that the orthogonality condition

$$
l^{i} m_{i}=0
$$

is met. The corresponding deformation gradient

$$
\boldsymbol{F}=\boldsymbol{I}+\left(l^{j} \boldsymbol{a}_{j}\right) \otimes\left(m_{i} \boldsymbol{a}^{i}\right)
$$

represents crystallographic slip on the plane normal to $m_{i} \boldsymbol{a}^{i}$ in the direction $l^{j} \boldsymbol{a}_{j}$.

\subsection{Harmonic lattice statics}

Next, we regard a crystal in its undeformed configuration as a collection of atoms whose nuclei occupy the sites $\boldsymbol{x}(\boldsymbol{l})$ of a simple Bravais lattice. By the Born-Oppenheimer approximation (Born and Oppenheimer 1927), the energy of the crystal can be written as

$$
\varepsilon=\phi\left(\left\{x(l), l \in Z^{3}\right\}\right) .
$$

The equilibrium positions of the atoms under the influence of applied forces $\boldsymbol{F}(\boldsymbol{l})$ are solutions of the equation

$$
-\frac{\partial \phi}{\partial \boldsymbol{x}(l)}+\boldsymbol{F}(\boldsymbol{l})=0 \quad l \in Z^{3} .
$$

The set of linear orthogonal transformations $Q: R^{3} \rightarrow R^{3}$ which bring the lattice into coincidence with itself forms a group $\mathcal{S}$ under composition called the symmetry group of the lattice. The symmetry group leaves the energy of a lattice invariant, i.e. expression (9) is invariant under the action of $Q \in \mathcal{S}$.

Next, the harmonic approximation is made in order to exploit powerful analytical tools such as Fourier methods. We let

$$
y(l)=x(l)+u(l)
$$

denote the deformed position of the atoms, where $\boldsymbol{u}(\boldsymbol{l})$ is the displacement of atom $\boldsymbol{l}$. A Taylor expansion of (10) retaining only the linear term in $\boldsymbol{u}(\boldsymbol{l})$ results in

$$
\sum_{l^{\prime}} \Phi_{i k}\left(l, l^{\prime}\right) u_{k}\left(l^{\prime}\right)=F_{i}(l)
$$


where the tensors

$$
\Phi_{i k}\left(\boldsymbol{l}, \boldsymbol{l}^{\prime}\right)=\left[\frac{\partial^{2} \phi}{\partial y_{i}(\boldsymbol{l}) \partial y_{k}\left(\boldsymbol{l}^{\prime}\right)}\right]_{u=0}
$$

are the stiffness coefficients or 'force constants', of the harmonic lattice. The force constant tensors satisfy additional relations that reflect properties of crystals such as translational invariance, symmetry group restrictions and the invariance of the energy density under rigid translations and rotations (see Born and Huang 1954). A useful consequence of translational invariance is the dependence of the force constants on the relative positions of the atoms alone, i.e.

$$
\Phi_{i j}\left(\boldsymbol{l}, \boldsymbol{l}^{\prime}\right)=\Phi_{i j}\left(\boldsymbol{l}-\boldsymbol{l}^{\prime}\right) .
$$

The energy (9) of the crystal in the harmonic approximation is given by the quadratic form

$$
\varepsilon=\frac{1}{2} \sum_{\left(l, l^{\prime}\right)} \Phi_{i k}\left(\boldsymbol{l}-\boldsymbol{l}^{\prime}\right) u_{i}(\boldsymbol{l}) u_{k}\left(\boldsymbol{l}^{\prime}\right) .
$$

An equivalent expression for the energy (15) that is better suited for the calculations that follow may be

$$
\varepsilon=-\frac{1}{4} \sum_{\left(l-l^{\prime}\right)} \Phi_{i k}\left(l-l^{\prime}\right)\left[u_{i}(l)-u_{i}\left(l^{\prime}\right)\right]\left[u_{k}(l)-u_{k}\left(l^{\prime}\right)\right] .
$$

This expression is obtained from expression (15) using the invariance of the energy under rigid translations. We emphasize that expression (16) is a positive definite quadratic form and hence is a convex function of the atomic displacement and, therefore, is not invariant with respect to lattice-preserving deformations such as those described in section 2.1. Hence, the energy function has no local minima that correspond to lattice defects such as dislocations.

Force constant tensors are microscopic quantities and may be calculated directly if $\phi$ is known. Alternatively, they may be inferred from experimentally measured macroscopic properties such as the elastic moduli, thermal expansion coefficients and specific heat using relations that connect the force constants to these macroscopic properties. For example, the relationship between the elasticity tensor and the force constant tensor (cf Born and Huang 1964) is

$$
c_{i j k l}=\Gamma_{i k j l}+\Gamma_{k j i l}-\Gamma_{k l i j}
$$

where

$$
\Gamma_{i j k l}=-\frac{1}{2 V} \sum_{l} \Phi_{i j}(l) x_{k}(l) x_{l}(l) .
$$

\subsection{The discrete Fourier transform}

In this section we briefly describe the use of the discrete Fourier transform (DFT) - a powerful tool in the context of harmonic lattice statics. The DFT of a lattice function $f(l)$ is given by

$$
\hat{f}(\boldsymbol{k})=V \sum_{l} f(\boldsymbol{l}) \mathrm{e}^{-\mathrm{i} k \cdot x(l)} \quad \boldsymbol{k} \in B
$$

where $B$ is the first Brillouin zone of the crystal. The inverse DFT recovers the original function, i.e.

$$
f(l)=\frac{1}{(2 \pi)^{3}} \int_{B} \hat{f}(\boldsymbol{k}) \mathrm{e}^{\mathrm{i} k \cdot x(l)} \mathrm{d}^{3} k .
$$


The properties of the DFT include the Parseval identity

$$
V \sum_{\boldsymbol{l}} f(\boldsymbol{l}) g^{*}(\boldsymbol{l})=\frac{1}{(2 \pi)^{3}} \int_{B} \hat{f}(\boldsymbol{k}) \hat{g}^{*}(\boldsymbol{k}) \mathrm{d}^{3} k
$$

and the convolution theorem

$$
(\widehat{f * g})(\boldsymbol{k})=\hat{f}(\boldsymbol{k}) \hat{g}(\boldsymbol{k})
$$

where $f$ and $g$ are lattice functions and the convolution operator $*$ is defined as

$$
(f * g)(\boldsymbol{l})=V \sum_{\boldsymbol{l}^{\prime}} f\left(\boldsymbol{l}-\boldsymbol{l}^{\prime}\right) g\left(\boldsymbol{l}^{\prime}\right)
$$

A direct solution of (12) is obtained by using these identities since the left-hand side of this expression is in convolution form. The DFT of (12) produces a system of three linear equations

$$
D_{i k}(\boldsymbol{k}) \hat{u}_{k}(\boldsymbol{k})=\hat{f}_{i}(\boldsymbol{k}) \quad \boldsymbol{k} \in B
$$

where

$$
D_{i k}(\boldsymbol{k})=\frac{1}{V^{2}} \hat{\Phi}_{i k}(\boldsymbol{k})
$$

is the dynamical matrix of the lattice and

$$
f_{i}(l)=\frac{1}{V} F_{i}(l)
$$

The transformed version of the lattice displacements now follows from (24) as

$$
\hat{u}_{k}(\boldsymbol{k})=D_{k i}^{-1}(\boldsymbol{k}) \hat{f}_{i}(\boldsymbol{k}) \quad \boldsymbol{k} \in B
$$

and the inverse DFT (20) provides the lattice displacements

$$
u_{k}(\boldsymbol{l})=\frac{1}{(2 \pi)^{3}} \int_{B} D_{k i}^{-1}(\boldsymbol{k}) \hat{f}_{i}(\boldsymbol{k}) \mathrm{e}^{\mathrm{i} k \cdot x(l)} \mathrm{d}^{3} k
$$

which furnishes the solution of (12) up to quadratures.

The integrals over the Brillouin zone $B$ such as those involved in the evaluation of the inverse DFT (20) may be computed by using the representations

$$
\boldsymbol{x}=l^{i} \boldsymbol{a}_{i} \quad \boldsymbol{k}=m_{i} \boldsymbol{a}^{i}
$$

in terms of the lattice and dual bases, respectively, whereupon (20) may be expressed in the form

$$
f(\boldsymbol{l})=\frac{1}{V} \frac{1}{(2 \pi)^{3}} \int_{0}^{2 \pi} \int_{0}^{2 \pi} \int_{0}^{2 \pi} \hat{f}(\boldsymbol{m}) \mathrm{e}^{\mathrm{i} m \cdot l} \mathrm{~d} m_{1} \mathrm{~d} m_{2} \mathrm{~d} m_{3} .
$$

The numerical evaluation of these integrals is straightforward for small values of the lattice indices $\left(l^{1}, l^{2}, l^{3}\right)$ while large values lead to highly oscillatory integrands whose evaluation requires special techniques (Gallego and Ortiz 1993).

\section{Eigendistorsions and dislocations in lattices}

In reality, the total energy (9) of a crystal is a non-convex function of the atomic displacements which allows for the emergence of defects such as dislocations. A conspicuous deficiency of the harmonic approximation, which would render it an unsuitable framework for the study of dislocations, is that (15) is a convex function, as already noted. Recourse to Mura's theory of eigendistorsions (Mura 1987, Gallego and Ortiz 1993) lifts this limitation. 
To adapt the harmonic theory to include eigendistorsions, we begin with the definition of a lattice deformation $\boldsymbol{\beta}\left(\boldsymbol{l}, \boldsymbol{l}^{\prime}\right)$ as a two-point function on the lattice $\left(\boldsymbol{\beta} \in R^{3}\right)$. Further, a lattice deformation field is said to be compatible if there exists a displacement field $\boldsymbol{u}(\boldsymbol{l})$ such that $\boldsymbol{\beta}\left(\boldsymbol{l}, \boldsymbol{l}^{\prime}\right)=\boldsymbol{u}(\boldsymbol{l})-\boldsymbol{u}\left(\boldsymbol{l}^{\prime}\right)$. A necessary and sufficient condition for a given lattice strain deformation to be compatible is

$$
\boldsymbol{\beta}\left(\boldsymbol{l}, \boldsymbol{l}^{\prime}\right)=\boldsymbol{\beta}\left(\boldsymbol{l}, \boldsymbol{l}^{\prime \prime}\right)-\boldsymbol{\beta}\left(\boldsymbol{l}^{\prime}, \boldsymbol{l}^{\prime \prime}\right) \quad \forall \boldsymbol{l}^{\prime \prime} \in Z^{3} .
$$

The energy of a compatible lattice strain field may be obtained from (16) as

$$
\varepsilon=-\frac{1}{4} \sum_{l, l^{\prime}} \Phi_{i k}\left(\boldsymbol{l}-\boldsymbol{l}^{\prime}\right) \beta_{i}\left(\boldsymbol{l}, \boldsymbol{l}^{\prime}\right) \beta_{k}\left(\boldsymbol{l}, \boldsymbol{l}^{\prime}\right) .
$$

Dislocations may be modelled in harmonic theory using incompatible lattice deformations $\beta^{\mathrm{E}}\left(\boldsymbol{l}, \boldsymbol{l}^{\prime}\right)$ called eigendistorsions. The strategy is to define eigendistorsions that correspond to dislocations and find the displacement fields and energies associated with these eigendistorsions. The energy of the crystal with defects is given by

$\varepsilon=-\frac{1}{4} \sum_{l, l^{\prime}} \Phi_{i k}\left(\boldsymbol{l}-\boldsymbol{l}^{\prime}\right)\left(u_{i}(\boldsymbol{l})-u_{i}\left(\boldsymbol{l}^{\prime}\right)-\beta_{i}^{\mathrm{E}}\left(\boldsymbol{l}, \boldsymbol{l}^{\prime}\right)\right)\left(u_{k}(\boldsymbol{l})-u_{k}\left(\boldsymbol{l}^{\prime}\right)-\beta_{k}^{\mathrm{E}}\left(\boldsymbol{l}, \boldsymbol{l}^{\prime}\right)\right)$.

The energy-wells (33) defined by the eigendistorsions, in addition to the usual well defined by (16), brings about the necessary non-convexity of energy. Given a superposed uniform macroscopic deformation and a choice of energy wells, corresponding to different eigendistorsions and the defect-free lattice, the preferred well is that which results in the least energy. For a fixed well, this criterion determines the range of uniform deformations, or applied stresses, for which the corresponding defect can be sustained stably within the lattice. We emphasize that the incompatible eigendistorsions are chosen to correspond to lattice preserving deformations.

To cement these ideas, we consider a square lattice with lattice parameter $b$ and nearest neighbour interactions. The coordinates of any atom in the lattice may be specified using two integers $\left(l^{1}, l^{2}\right)$. We investigate the 'rigid sliding' motion of this crystal, in which the part of the crystal with $l^{2}>0$ (the top half) is rigidly displaced in the 1 direction with respect to the bottom half by a distance $x$. It is clear that when $x=b$, the energy of the crystal must be zero, as the deformation is lattice preserving. Although the energy computed using (16) does not vanish at this value of $x$, that computed using (33) does vanish when the eigendistorsions are chosen as

$\beta_{i}^{\mathrm{E}}\left(\boldsymbol{l}, \boldsymbol{l}^{\prime}\right)=b \delta_{i 1} \sum_{M=-\infty}^{\infty}\left(\delta\left(l^{2}-1\right) \delta\left(l^{\prime 2}\right)-\delta\left(l^{2}\right) \delta\left(l^{\prime 2}-1\right)\right) \delta\left(l^{1}-M\right) \delta\left(l^{\prime 1}-M\right)$.

For small values of $x$ the energy expression described by (16) is valid, it breaks down only for large values of $x$. In fact, expression (16) is meaningful as long as $x \leqslant b / 2$, while the energy-well defined by (33) becomes operative for larger values of $x(x \geqslant b / 2)$. This illustration elucidates two essential points. First, is the importance of the correct definition of the eigendistorsions. Second, it emphasizes the role of choosing the correct energy-well; in the case just discussed the energy-well defined by the eigendistorsions becomes operative only after $x>b / 2$. If this condition is not satisfied the energy should be computed using the well defined by (16).

We now turn to the definition of the eigendistorsions required in the study of dislocation loops. We consider a lattice basis such that $l^{3}=$ constant defines the slip plane. To define the eigendistorsions corresponding to a dislocation loop, we first define a unit eigendistorsion or 
loopon, i.e. when slip occurs only at one atomic site on the slip plane, say at $M=\left(M^{1}, M^{2}, 0\right)$, denoted by $\boldsymbol{\beta}^{\mathrm{EU}}\left(\boldsymbol{l}-\boldsymbol{M}, \boldsymbol{l}^{\prime}-\boldsymbol{M}\right)$, as

$$
\beta^{\mathrm{EU}}\left(\boldsymbol{l}-\boldsymbol{M}, \boldsymbol{l}^{\prime}-\boldsymbol{M}\right)=\boldsymbol{b} f\left(\boldsymbol{l}-\boldsymbol{M}, \boldsymbol{l}^{\prime}-\boldsymbol{M}\right)
$$

where $\boldsymbol{b}$ is the Burgers vector. The specific details of the definition of $f$ will depend on the crystallography and these will be discussed in later sections for specific crystals; presently we assume that $\boldsymbol{\beta}^{\mathrm{EU}}$ is defined in order to keep to a general discussion. The eigendistorsion of a dislocation loop is now taken as the superposition of loopons, i.e.

$$
\boldsymbol{\beta}^{\mathrm{EU}}\left(\boldsymbol{l}, \boldsymbol{l}^{\prime}\right)=\sum_{M \in \text { loop }} \beta^{\mathrm{EU}}\left(\boldsymbol{l}-\boldsymbol{M}, \boldsymbol{l}^{\prime}-\boldsymbol{M}\right) .
$$

Minimizing the energy function (3) with respect to $\boldsymbol{u}(\boldsymbol{l})$ gives the equilibrium lattice displacements corresponding to the defects described by $\boldsymbol{\beta}^{\mathrm{E}}\left(\boldsymbol{l}, \boldsymbol{l}^{\prime}\right)$. A set of linear equations for the energy minimizing displacements $\boldsymbol{u}(\boldsymbol{l})$ are obtained in the form:

$$
\sum_{l^{\prime}} \Phi_{i k}\left(\boldsymbol{l}-\boldsymbol{l}^{\prime}\right) u_{k}\left(\boldsymbol{l}^{\prime}\right)=F_{i}^{\mathrm{E}}(\boldsymbol{l})
$$

where

$$
F_{i}^{\mathrm{E}}(\boldsymbol{l})=\sum_{\boldsymbol{l}^{\prime}} \Phi_{i k}\left(\boldsymbol{l}-\boldsymbol{l}^{\prime}\right) \beta_{k}^{\mathrm{E}}\left(\boldsymbol{l}^{\prime}, \boldsymbol{l}\right)
$$

is defined as the eigenforce field. The associated energy may be computed from (33) as

$$
\varepsilon=-\frac{1}{4} \sum_{l-l^{\prime}} \Phi_{i k}\left(\boldsymbol{l}-\boldsymbol{l}^{\prime}\right) \beta_{i}^{\mathrm{E}}\left(\boldsymbol{l}, \boldsymbol{l}^{\prime}\right) \beta_{k}^{\mathrm{E}}\left(\boldsymbol{l}, \boldsymbol{l}^{\prime}\right)-\frac{1}{2} \sum_{l} F_{i}^{\mathrm{E}}(\boldsymbol{l}) u_{i}(\boldsymbol{l}) .
$$

As discussed in section 2.3, the DFT of the solution of (37) is obtained as

$$
\hat{u}_{k}(\boldsymbol{k})=D_{k i}^{-1}(\boldsymbol{k}) \hat{f}_{i}^{\mathrm{E}}(\boldsymbol{k})
$$

where

$$
f_{i}^{\mathrm{E}}(\boldsymbol{l})=\frac{1}{V} F_{i}^{\mathrm{E}}(\boldsymbol{l})
$$

and the lattice displacements are recovered by application of the inverse DFT (23), with the result that

$$
u_{k}(l)=\frac{1}{(2 \pi)^{3}} \int_{B} D_{k i}^{-1}(\boldsymbol{k}) \hat{f}_{i}^{\mathrm{E}}(\boldsymbol{k}) \mathrm{e}^{\mathrm{i} k \cdot x(l)} \mathrm{d}^{3} k .
$$

Now, by properties (21) and (22) of the DFT, the energy (39) may be computed as

$$
\varepsilon=-\frac{1}{4} \sum_{l, l^{\prime}} \Phi_{i k}\left(\boldsymbol{l}-\boldsymbol{l}^{\prime}\right) \beta_{i}^{\mathrm{E}}\left(\boldsymbol{l}, \boldsymbol{l}^{\prime}\right) \beta_{k}^{\mathrm{E}}\left(\boldsymbol{l}, \boldsymbol{l}^{\prime}\right)-\frac{1}{(2 \pi)^{3}} \int_{B} \frac{1}{2} \hat{f}_{i}^{\mathrm{E}}(\boldsymbol{k}) \hat{u}_{i}(\boldsymbol{k}) \mathrm{d}^{3} k .
$$

Finally, insertion of (40) into (43) gives

$\varepsilon=-\frac{1}{4} \sum_{l, l^{\prime}} \Phi_{i k}\left(\boldsymbol{l}-\boldsymbol{l}^{\prime}\right) \beta_{i}^{\mathrm{E}}\left(\boldsymbol{l}, \boldsymbol{l}^{\prime}\right) \beta_{k}^{\mathrm{E}}\left(\boldsymbol{l}, \boldsymbol{l}^{\prime}\right)-\frac{1}{(2 \pi)^{3}} \int_{B} \frac{1}{2} D_{k i}^{-1}(\boldsymbol{k}) \hat{f}_{k}^{\mathrm{E} *}(\boldsymbol{k}) \hat{f}_{i}^{\mathrm{E}}(\boldsymbol{k}) \mathrm{d}^{3} k$.

which provides, up to quadratures, an explicit analytic expression for the energy of the dislocation loop.

For the solution to be valid, the displacements given by equation (42) must satisfy a condition similar to the one described before. The specific condition as to when the eigendistorsion energy-well becomes operative will depend on the crystallography and also on the nature of the dislocations studied. If it is found that the displacements given by (42) do not satisfy the condition, it implies that the dislocation loop is not stable. In such cases the 
condition is enforced by superposing on the crystal a homogeneous deformation that causes the eigendistorsion energy-well to become operative. Physically, this means that an external stress is required for the formation of the dislocations - they will not be spontaneously nucleated. The total potential energy, i.e. including the interaction of the loop with the externally applied stress, may be computed as

$$
\begin{aligned}
\varepsilon=-\frac{1}{4} \sum_{l, l^{\prime}} & \Phi_{i k}\left(\boldsymbol{l}-\boldsymbol{l}^{\prime}\right) \beta_{i}^{\mathrm{E}}\left(\boldsymbol{l}, \boldsymbol{l}^{\prime}\right) \beta_{k}^{\mathrm{E}}\left(\boldsymbol{l}, \boldsymbol{l}^{\prime}\right)-\frac{1}{(2 \pi)^{3}} \int_{B} \frac{1}{2} D_{k i}^{-1}(\boldsymbol{k}) \hat{f}_{k}^{\mathrm{E} *}(\boldsymbol{k}) \hat{f}_{i}^{\mathrm{E}}(\boldsymbol{k}) \mathrm{d}^{3} k \\
& -\sum_{l} F_{i}^{\mathrm{E}}(\boldsymbol{l}) u_{i}^{\mathrm{S}}(\boldsymbol{l})
\end{aligned}
$$

where $u_{i}^{\mathrm{S}}$ is the displacement field due to the homogeneous applied stress and $\varepsilon$ is the excess potential energy over and above the potential energy of the homogeneously strained crystal.

In order to estimate the probability of spontaneous nucleation of a dislocation loop, the energy barriers which oppose the growth of the loop need to be computed. To this end, we consider two dislocation loops whose domains differ by a single site $\left(L^{1}, L^{2}, 0\right)$. Two cases may be considered separately; (1) when the loop does not contain the site $\left(L^{1}, L^{2}, 0\right)$ with potential energy $\varepsilon_{1}$ and displacements $\boldsymbol{u}^{1}(\boldsymbol{l})$, and (2) when the loop has 'grown' to include the site $\left(L^{1}, L^{2}, 0\right)$ with energy $\varepsilon_{2}$ and displacements $\boldsymbol{u}^{2}(\boldsymbol{l})$. It is clear that configuration 2 is obtained from configuration 1 by imposing a loop on $\beta^{\mathrm{EU}}$ at the site $\left(L^{1}, L^{2}, 0\right)$. Both these configurations correspond to local minima in energy as shown schematically in figure 1 , and the path traversed in going from configuration 1 to 2 is parametrized by a number $\lambda$, where $\lambda=0$ corresponds to the energy minimum of 1 , while $\lambda=1$ implies configuration 2 . The problem is to find the value $\lambda_{\mathrm{S}}$ which corresponds to the saddle point equilibrium. To compute $\lambda_{\mathrm{S}}$, we note that the potential energy (measured with respect to the homogeneously deformed crystal) in the well corresponding to configuration 1 is given as (using (45))

$$
E_{1}(\lambda)=\varepsilon_{1}+\frac{\lambda^{2}}{2} \sum_{l} \boldsymbol{F}^{\mathrm{EU}}(\boldsymbol{l}) \cdot \boldsymbol{u}^{\mathrm{U}}(\boldsymbol{l})
$$

where $\boldsymbol{F}^{\mathrm{EU}}(\boldsymbol{l})$ and $\boldsymbol{u}^{\mathrm{U}}(\boldsymbol{l})$ are the eigenforces and displacements due to the presence of a loopon $\beta^{\mathrm{EU}}$ at $\left(L^{1}, L^{2}, 0\right)$. Similarly, the equation of the potential energy (again, measured with respect to the homogeneous deformed crystal using (45)) from well 2 may be put down as

$$
E_{2}(\lambda)=\varepsilon_{2}+\frac{(1-\lambda)^{2}}{2} \sum_{l} \boldsymbol{F}^{\mathrm{EU}}(\boldsymbol{l}) \cdot \boldsymbol{u}^{\mathrm{U}}(\boldsymbol{l}) .
$$

At the saddle point $\left(\lambda=\lambda_{\mathrm{S}}\right)$, we have that $E_{i}\left(\lambda_{\mathrm{S}}\right)=E_{2}\left(\lambda_{\mathrm{S}}\right)$ or

$$
\lambda_{\mathrm{S}}=\frac{\varepsilon_{2}-\varepsilon_{1}+(1 / 2) \Sigma_{l} \boldsymbol{F}^{\mathrm{EU}}(\boldsymbol{l}) \cdot \boldsymbol{u}^{\mathrm{U}}(\boldsymbol{l})}{\Sigma_{l} \boldsymbol{F}^{\mathrm{EU}}(\boldsymbol{l}) \cdot \boldsymbol{u}^{\mathrm{U}}(\boldsymbol{l})} .
$$

If the computed value of $\lambda_{\mathrm{S}}$ satisfies $0<\lambda_{\mathrm{S}}<1$ then well 2 is accessible from well 1 (via the path parametrized by $\lambda$ ) and the activation energy of this process is given by

$$
\varepsilon^{\mathrm{a}}=\frac{\lambda_{\mathrm{S}}^{2}}{2} \sum_{l} \boldsymbol{F}^{\mathrm{EU}}(\boldsymbol{l}) \cdot \boldsymbol{u}^{\mathrm{U}}(\boldsymbol{l}) .
$$

If $\lambda_{\mathrm{S}}<0$, then the loop will spontaneously grow (no activation barrier) and if $\lambda_{\mathrm{S}}>1$ the path parametrized by $\lambda$ is not physically realizable. Thus, on specifying the eigendistorsions of a loop, the energy, displacements and the activation energies for formation and growth of the loop may be computed. 


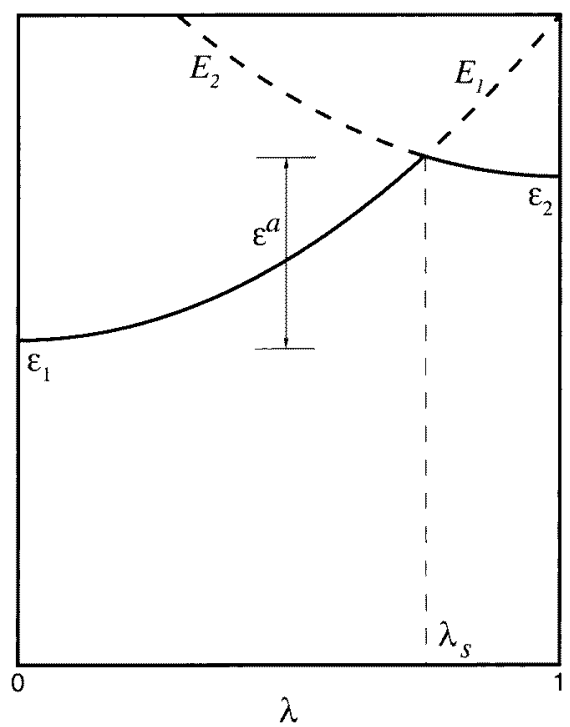

Figure 1. Schematic depiction of the activation barrier for the growth of a dislocation loop.

\section{Screw dislocation dipole in a square lattice}

As a first illustration of the theory, we treat the case of a screw dislocation dipole in a square lattice. It is also our intention to compare the results obtained from the harmonic theory to those of continuum linear elasticity.

In the case of the square lattice, $B=[-\pi / a, \pi / a]^{2}$, where $a$ is the lattice parameter. The force constants are

$$
\Phi_{33}(l)= \begin{cases}-\mu a & \left|l^{1}\right|+\left|l^{2}\right|=1 \\ 4 \mu a & l=0 \\ 0 & \text { otherwise }\end{cases}
$$

where $\mu$ is the shear modulus. The sole non-zero component of the dynamical matrix is

$$
D_{33}=\frac{4 \mu}{a}\left(\sin ^{2} \frac{k_{1} a}{2}+\sin ^{2} \frac{k_{2} a}{2}\right) .
$$

The loopon (at $(0,0,0))$ in the case of the square lattice is defined as

$$
\beta^{\mathrm{EU}}\left(\boldsymbol{l}, \boldsymbol{l}^{\prime}\right)=\boldsymbol{b}\left(\delta\left(l^{2}-1\right) \delta\left(l^{\prime 2}\right)-\delta\left(l^{2}\right) \delta\left(l^{\prime 2}-1\right)\right) \delta\left(l^{1}\right) \delta\left(l^{\prime 1}\right)
$$

where $\boldsymbol{b}=a(0,0,1)$ is the Burgers vector. We construct the dislocation dipole by placing two dislocations of opposite sign $N$ lattice cells apart, where we take $N$ to be odd $(N=2 n+1)$. Here and henceforth $\delta(l)$ represents the discrete delta function, i.e. $\delta(l)=1, l=0$ and $\delta(l)=0, l \neq 0$. On choosing the origin as the dipole centre and taking the plane $x_{2}=0$ as the cut plane, the eigendistorsions are

$$
\beta^{\mathrm{EU}}\left(\boldsymbol{l}, \boldsymbol{l}^{\prime}\right)=\sum_{M^{1}=-n, n ; M^{2}=0} \beta^{\mathrm{EU}}\left(\boldsymbol{l}-\boldsymbol{M}, \boldsymbol{l}^{\prime}-\boldsymbol{M}\right)
$$

and the resulting eigenforces are found to be

$$
\hat{f}_{3}^{\mathrm{E}}(\boldsymbol{k})=2 \mathrm{i} \mu a^{2} \frac{\sin \left(k_{2} a / 2\right)}{\sin \left(k_{1} a / 2\right)} \sin \frac{k_{1} r}{2}
$$


where $r=N a$ is the length of the dipole. From (40), the DFT of the corresponding displacement field is found to be

$$
\hat{u}_{3}(\boldsymbol{k})=\frac{\mathrm{i} a^{3}}{2}\left(\sin ^{2} \frac{k_{1} a}{2}+\sin ^{2} \frac{k_{2} a}{2}\right)^{-1} \frac{\sin \left(k_{2} a / 2\right)}{\sin \left(k_{1} a / 2\right)} \sin \frac{k_{1} r}{2} .
$$

The continuum limit is realized by formally letting $a \rightarrow 0$, hence

$$
\hat{u}_{3}(\boldsymbol{k}) \sim a \frac{k_{2}}{k_{1}} \frac{1}{k^{2}}\left(\mathrm{e}^{\mathrm{i} k_{1} r / 2}-\mathrm{e}^{\mathrm{i} k_{1} r / 2}\right) .
$$

The inverse Fourier transform of the function $\left(a / k^{2}\right)\left(k_{2} / k_{1}\right)$ is $a \theta /(2 \pi)$ and the displacement field (56) is that of two linear elastic screw dislocations of opposite sign and Burgers vector $a$ at $x_{1}= \pm r / 2$. Relation (44) provides the energy per unit length of the dislocation to be

$$
\begin{aligned}
\frac{\varepsilon}{L}=\frac{1}{2} \mu a r- & \frac{\mu a^{4}}{4 \pi^{2}} \int_{-\pi / a}^{\pi / a} \int_{-\pi / a}^{\pi / a} \frac{1}{2}\left(\sin ^{2} \frac{k_{1} a}{2}+\sin ^{2} \frac{k_{2} a}{2}\right)^{-1} \\
& \times\left(\frac{\sin \left(k_{2} a / 2\right)}{\sin \left(k_{1} a / 2\right)} \sin \frac{k_{1} r}{2}\right)^{2} \mathrm{~d} k_{1} \mathrm{~d} k_{2} .
\end{aligned}
$$

Integration with respect to $k_{2}$ explicitly leaves

$$
\frac{\varepsilon}{L}=C_{N} \mu a^{2}
$$

where

$C_{N}=\frac{N}{2}-\frac{1}{4 \pi} \int_{-\pi}^{\pi}\left(1+\csc ^{2} \frac{\xi}{2}-\left|\csc \frac{\xi}{2}\right| \sqrt{1+\sin ^{2} \frac{\xi}{2}}\right) \frac{\sin ^{2}(\xi N / 2)}{1+\sin ^{2}(\xi / 2)} \mathrm{d} \xi$.

The large $N$ behaviour of (58) corresponds to the continuum limit. The result of this analysis is

$$
\frac{\varepsilon}{L} \sim \frac{\mu a^{2}}{2 \pi} \log \frac{r}{r_{0}}
$$

where

$$
r_{0}=\frac{a}{\pi} \mathrm{e}^{-\gamma} \approx 0.178718 a
$$

is the dislocation core cut-off radius predicted by lattice theory.

The preceding example illustrates how consideration of the discreteness of the lattice eliminates the divergence at the dislocation core and determines a precise value of the core cut-off radius $r_{0}$. Table 1 displays a comparison of the energies of small screw dipoles computed via lattice theory and linear elasticity. It is seen that the discrepancies are large for small dipoles.

Table 1. Energies of a screw dislocation dipole in a square lattice.

\begin{tabular}{llll}
\hline$N$ & $C_{N}$ & Continuum & Error $(\%)$ \\
\hline 0 & 0 & $-\infty$ & - \\
1 & $1 / 4$ & 0.274056 & 9.62 \\
3 & 0.430281 & 0.448906 & 4.32 \\
5 & 0.512902 & 0.530206 & 3.37 \\
7 & 0.566760 & 0.583757 & 3.00 \\
9 & 0.606874 & 0.623755 & 2.78 \\
\hline
\end{tabular}




\section{Application to nascent dislocation loops in FCC crystals}

In this section, we apply the tools of analysis developed in the foregoing to the analytical characterization of the structure and energies of nascent dislocation loops in some FCC crystals.

\subsection{FCC lattice model}

Let $a$ be the lattice parameter of the FCC lattice. One possible choice of the lattice basis is

$$
\boldsymbol{a}_{1}=(a / 2)(0,1,1) \quad \boldsymbol{a}_{2}=(a / 2)(1,0,1) \quad \boldsymbol{a}_{3}=(a / 2)(1,1,0)
$$

where all components are relative to an orthonormal Cartesian frame coincident with the cubic directions of the lattice. The dual basis is

$\boldsymbol{a}^{1}=(1 / a)(-1,1,1) \quad \boldsymbol{a}^{2}=(1 / a)(1,-1,1) \quad \boldsymbol{a}^{3}=(1 / a)(1,1,-1)$.

The unit cell volume is $V=a^{3} / 4$.

We consider a simple model of FCC with interaction restricted to the 12 nearest neighbours. The lattice and Cartesian coordinates of an atom at the origin and its 12 neighbours are collected in table 2. The geometry of the FCC stencil is shown in figure 2. These assumptions and imposition of the cubic symmetry requires that the most general form of the force constant tensors is given as (Begbie 1947)

$$
\begin{array}{ll}
\Phi(1,0,0)=-\left(\begin{array}{lll}
\beta & 0 & 0 \\
0 & \alpha & \gamma \\
\gamma & 0 & \alpha
\end{array}\right) & \Phi(0,1,0)=-\left(\begin{array}{ccc}
\alpha & 0 & \gamma \\
0 & \beta & 0 \\
0 & \gamma & \alpha
\end{array}\right) \\
\Phi(0,0,1)=-\left(\begin{array}{lll}
\alpha & \gamma & 0 \\
\gamma & \alpha & 0 \\
0 & 0 & \beta
\end{array}\right) & \Phi(0,-1,1)=-\left(\begin{array}{ccc}
\beta & 0 & 0 \\
0 & \alpha & -\gamma \\
0 & -\gamma & \alpha
\end{array}\right) \\
\Phi(1,0,-1)=-\left(\begin{array}{ccc}
\alpha & 0 & -\gamma \\
0 & \beta & 0 \\
-\gamma & 0 & \alpha
\end{array}\right) & \Phi(-1,1,0)=-\left(\begin{array}{ccc}
\alpha & -\gamma & 0 \\
-\gamma & \alpha & 0 \\
0 & 0 & \beta
\end{array}\right)
\end{array}
$$

for some constants $\alpha, \beta$ and $\gamma$. The remaining force constant tensors follow from the relation

$$
\Phi_{i j}(-l)=\Phi_{i j}(l)
$$

and invariance of the energy under rigid translations implies

$$
\Phi(0,0,0)=-\sum_{l \neq 0} \Phi(l)=4(2 \alpha+\beta)\left(\begin{array}{ccc}
1 & 0 & 0 \\
0 & 1 & 0 \\
0 & 0 & 1
\end{array}\right) .
$$

The relation between elastic constants and the force constants (17) may now be used to determine the constants $\alpha, \beta$ and $\gamma$ in terms of the elastic constants $c_{11}, c_{12}$ and $c_{44}$ (in Voigt notation). The calculation reveals the following relations

$$
\alpha=\frac{a}{4} c_{11} \quad \beta=\frac{a}{4}\left(2 c_{44}-c_{11}\right) \quad \gamma=\frac{a}{4}\left(c_{12}+c_{44}\right)
$$

which permit the identification of the force constants of the lattice from elastic moduli data. A few selected examples are collected in table 3 .

A note on the correctness of our nearest neighbour model is necessary. The force constants may be computed directly from, say, an embedded atom method (EAM) potential. In the case of Au the values obtained using EAM potentials (Oh and Johnson 1988) are $0.558,-0.174$ and 0.673 for $\alpha, \beta$ and $\gamma$, respectively (compare with table 3 ), while those of $\mathrm{Cu}$ obtained from the same potentials are $0.933,-0.103$ and 1.012 which are closed to those in table 3 . In cases where the nearest neighbour model is not satisfactory, the formulation may be generalized to 


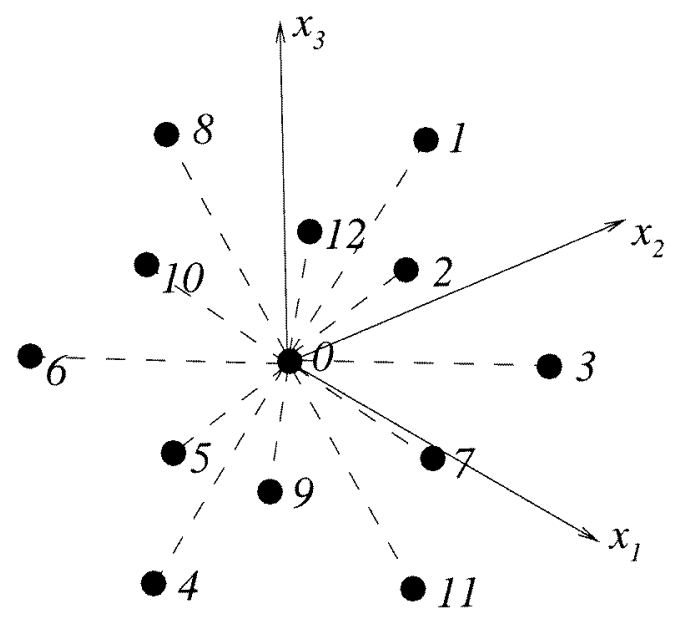

Figure 2. Stencil geometry for FCC crystals.

Table 2. Cartesian and lattice coordinates of interacting atoms in the model of a FCC lattice.

\begin{tabular}{ccllrrr}
\hline Atom & $x^{1}$ & $x^{2}$ & $x^{3}$ & $l^{1}$ & $l^{2}$ & $l^{3}$ \\
\hline 0 & 0 & 0 & 0 & 0 & 0 & 0 \\
1 & 0 & $a / 2$ & $a / 2$ & 1 & 0 & 0 \\
2 & $a / 2$ & 0 & $a / 2$ & 0 & 1 & 0 \\
3 & $a / 2$ & $a / 2$ & 0 & 0 & 0 & 1 \\
4 & 0 & $-a / 2$ & $-a / 2$ & -1 & 0 & 0 \\
5 & $-a / 2$ & 0 & $-a / 2$ & 0 & -1 & 0 \\
6 & $-a / 2$ & $-a / 2$ & 0 & 0 & 0 & -1 \\
7 & 0 & $a / 2$ & $a / 2$ & 0 & -1 & 1 \\
8 & $-a / 2$ & 0 & $a / 2$ & 1 & 0 & -1 \\
9 & $a / 2$ & $-a / 2$ & 0 & -1 & 1 & 0 \\
10 & 0 & $-a / 2$ & $a / 2$ & 0 & 1 & -1 \\
11 & $a / 2$ & 0 & $-a / 2$ & -1 & 0 & 1 \\
12 & $-a / 2$ & $a / 2$ & 0 & 1 & -1 & 0 \\
\hline
\end{tabular}

Table 3. Elastic moduli $\left(\mathrm{eV} \AA^{-3}\right)$, lattice parameters $(\AA)$ and force constants $\left(\mathrm{eV} \AA^{-2}\right)$ of selected FCC crystals.

\begin{tabular}{llllllll}
\hline Material & $c_{11}$ & $c_{12}$ & $c_{44}$ & $a$ & $\alpha$ & \multicolumn{1}{l}{$\beta$} & $\gamma$ \\
\hline $\mathrm{Ag}$ & 0.759 & 0.564 & 0.282 & 4.084 & 0.775 & -0.199 & 0.864 \\
$\mathrm{Al}$ & 0.737 & 0.389 & 0.229 & 4.032 & 0.743 & -0.281 & 0.623 \\
$\mathrm{Au}$ & 1.156 & 0.976 & 0.261 & 4.079 & 1.179 & -0.646 & 1.261 \\
$\mathrm{Cu}$ & 1.032 & 0.745 & 0.470 & 3.615 & 0.933 & -0.084 & 1.098 \\
$\mathrm{Ni}$ & 1.559 & 0.932 & 0.768 & 3.523 & 1.373 & -0.021 & 1.497 \\
$\mathrm{~Pb}$ & 0.309 & 0.263 & 0.093 & 4.948 & 0.382 & -0.151 & 0.441 \\
$\mathrm{Th}$ & 0.468 & 0.304 & 0.297 & 5.091 & 0.596 & 0.161 & 0.765 \\
\hline
\end{tabular}

include higher neighbours, and the force constant matrix may be obtained directly from the interatomic potential. 
For the FCC lattice model presented here, the dynamical matrix is computed to be

$$
\begin{aligned}
D_{11}= & \frac{4}{a^{2}}\left\{c_{11}\left[2-\cos \left(k_{1} a / 2\right)\left(\cos \left(k_{2} a / 2\right)+\cos \left(k_{3} a / 2\right)\right)\right]\right. \\
& \left.+\left(2 c_{44}-c_{11}\right)\left(1-\cos \left(k_{2} a / 2\right) \cos \left(k_{3} a / 2\right)\right)\right\}
\end{aligned}
$$

where the remaining components follow by permutation of the indices.

\subsection{Nascent loops in FCC crystals}

We define the loopon (unit eigendistorsion at $(0,0,0))$ as

$$
\begin{aligned}
\beta^{\mathrm{EU}}\left(\boldsymbol{l}, l^{\prime}\right)=\boldsymbol{b}[\delta & \left.\left(l^{1}\right) \delta\left(l^{2}\right) \delta\left(l^{3}-1\right) \delta\left(l^{11}\right) \delta\left(l^{\prime 2}\right) \delta\left(l^{\prime 3}\right)-\delta\left(l^{1}\right) \delta\left(l^{2}\right) \delta\left(l^{3}\right) \delta\left(l^{11}\right) \delta\left(l^{\prime 2}\right) \delta\left(l^{\prime 3}-1\right)\right] \\
& +\frac{\boldsymbol{b}}{2}\left[\delta\left(l^{1}\right) \delta\left(l^{2}\right) \delta\left(l^{3}-1\right) \delta\left(l^{1}-1\right) \delta\left(l^{\prime 2}\right) \delta\left(l^{\prime 3}\right)\right. \\
& \left.-\delta\left(l^{1}-1\right) \delta\left(l^{2}\right) \delta\left(l^{3}\right) \delta\left(l^{\prime 1}\right) \delta\left(l^{\prime 2}\right) \delta\left(l^{\prime 3}-1\right)\right] \\
& +\frac{\boldsymbol{b}}{2}\left[\delta\left(l^{1}\right) \delta\left(l^{2}\right) \delta\left(l^{3}-1\right) \delta\left(l^{11}\right) \delta\left(l^{\prime 2}-1\right) \delta\left(l^{\prime 3}\right)\right. \\
& \left.-\delta\left(l^{1}\right) \delta\left(l^{2}-1\right) \delta\left(l^{3}\right) \delta\left(l^{\prime 1}\right) \delta\left(l^{\prime 2}\right) \delta\left(l^{\prime 3}-1\right)\right]
\end{aligned}
$$

where $\boldsymbol{b}=(b / \sqrt{2})(011)$ is the Burgers vector $(b=(a / \sqrt{2}))$. The eigenforces corresponding to the loopon are

$$
\begin{aligned}
& \boldsymbol{F}^{\mathrm{EU}}(0,0,1)=-\boldsymbol{F}^{\mathrm{EU}}(0,0,0)=\frac{b}{2 \sqrt{2}}(\gamma, 3 \alpha+\beta-\gamma, 2 \alpha+2 \beta-\gamma) \\
& \boldsymbol{F}^{\mathrm{EU}}(-1,0,1)=-\boldsymbol{F}^{\mathrm{EU}}(1,0,0)=\frac{b}{2 \sqrt{2}}(-\gamma, \beta, \alpha) \\
& \boldsymbol{F}^{\mathrm{EU}}(0,-1,1)=-\boldsymbol{F}^{\mathrm{EU}}(0,1,0)=\frac{b}{2 \sqrt{2}}(0, \alpha-\gamma, \alpha-\gamma) \\
& \boldsymbol{F}^{\mathrm{EU}}(\boldsymbol{l})=0 \quad \text { for all other } \boldsymbol{l} .
\end{aligned}
$$

To verify that this definition of the unit eigendistorsion is meaningful, we construct an eigendistorsion field that represents rigid sliding of the top half of the crystal $\left(l^{3}>0\right)$ with respect to the bottom half $\left(l^{3} \leqslant 0\right)$ by a Burgers vector superposing the unit eigendistorsions. The resultant solution must reproduce the rigid sliding displacement and must have zero energy, as the deformation is a lattice preserving one. The eigendistorsion field corresponding to the rigid displacement is

$$
\beta^{\mathrm{E}}\left(\boldsymbol{l}, \boldsymbol{l}^{\prime}\right)=\sum_{M \in \text { slip plane }} \beta^{\mathrm{EU}}\left(\boldsymbol{l}-\boldsymbol{M}, \boldsymbol{l}^{\prime}-\boldsymbol{M}\right) .
$$

The resulting eigenforces are

$$
\boldsymbol{F}^{\mathrm{E}}\left(l^{1}, l^{2}, 1\right)=-\boldsymbol{F}^{\mathrm{E}}\left(l^{1}, l^{2}, 0\right)=(2 \alpha+\beta-\gamma) \boldsymbol{b}
$$

and vanish for all other $\boldsymbol{l}$. Solving for the displacements using (42) provides that $u(l)=(b / 2)$, $l^{3}>0$ and $u(l)=-(b / 2), l^{3} \leqslant 0$ which is the expected result. The energy evaluated using (44) vanishes establishing the correctness of the approach.

We now turn to the smallest loop, i.e. the loop defined by the loopon itself. To stabilize the loopon (i.e. to ensure that the energy-well defined by the eigendistorsions is valid), a homogeneous state of deformation is imposed on the crystal given by

$$
\boldsymbol{F}=\boldsymbol{I}+\eta \frac{1}{\sqrt{6}}[011] \otimes[11 \overline{1}]
$$


where $\eta$ is chosen such that the total displacements (the sum of those due to the homogeneous strain and those due to the eigendistorsions) satisfies

$$
(\boldsymbol{u}(0,0,1)-\boldsymbol{u}(0,0,0)) \cdot \hat{\boldsymbol{b}}=b / 2
$$

where $\hat{b}$ is a unit vector along $\boldsymbol{b}$ (this is similar to the condition discussed in section 3 ). The resolved shear stress $\tau$ on the slip plane is given by

$$
\tau=\mu \eta
$$

where $\mu$ is the effective shear modulus defined by

$$
\mu=\frac{c_{11}+c_{44}-c_{12}}{3} .
$$

The displacements due to the loopon may be evaluated using (42) and the expression for the potential energy (45) reduces to

$\varepsilon=\frac{b^{5}(5 \alpha+3 \beta-2 \gamma)}{8}-\frac{1}{(2 \pi)^{3}} \int_{B} \frac{1}{2} D_{k i}^{-1}(\boldsymbol{k}) \hat{f}_{k}^{\mathrm{EU} *}(\boldsymbol{k}) \hat{f}_{i}^{\mathrm{EU}}(\boldsymbol{k}) \mathrm{d}^{3} k-\sum_{\boldsymbol{l}} F_{i}^{\mathrm{E}}(\boldsymbol{l}) u_{i}^{\mathrm{S}}(\boldsymbol{l})$

where $u_{i}^{\mathrm{S}}$ is the displacement field due to the homogeneous deformation (77). To compute the activation energy for the formation of this loop, we take $\varepsilon_{1}=0$ and $\varepsilon_{2}=\varepsilon$ in (48) and obtain the activation energy from (49). The results of the calculation are tabulated in table 4.

Table 4. Potential energy (eV), activation energy $(\mathrm{eV})$ and the resolved shear stress required to stabilize the smallest loop in FCC crystals.

\begin{tabular}{lclll}
\hline Material & $\varepsilon$ & $\lambda_{\mathrm{S}}$ & $\varepsilon^{\mathrm{a}}$ & $\tau / \mu$ \\
\hline $\mathrm{Ag}$ & -1.277681 & -0.084895 & 0.0 & 0.542755 \\
$\mathrm{Al}$ & -1.334087 & -0.207294 & 0.0 & 0.528888 \\
$\mathrm{Au}$ & -2.561564 & -0.054895 & 0.0 & 0.570245 \\
$\mathrm{Cu}$ & -1.097950 & -0.056299 & 0.0 & 0.526683 \\
$\mathrm{Ni}$ & -1.575131 & -0.090753 & 0.0 & 0.513675 \\
$\mathrm{~Pb}$ & -1.052933 & -0.052122 & 0.0 & 0.568959 \\
$\mathrm{Th}$ & -1.535036 & -0.096367 & 0.0 & 0.543369 \\
\hline
\end{tabular}

It is easily seen that in the nearest neighbour model we have constructed here, the strength of the crystal $\tau_{\max }$ (the maximum possible resolved shear stress on the slip plane) is given by

$$
\tau_{\max }=\sqrt{\frac{3}{8}} \mu=0.612 \mu .
$$

In all the metals, the resolved shear stress required to stabilize the loops is smaller than $\tau_{\max }$ it is about $0.9 \tau_{\max }$. The value of $\lambda_{\mathrm{S}}$ is negative in all cases implying that nucleation of the loop is spontaneous at such high values of the applied stress. Note that the negative values of the potential energies indicate the reduction in its value with reference to the homogeneously strained state as is expected.

Next, we consider hexagonal loops in these crystals. The eigendistorsion for this case is obtained by superposing loopons placed at $(0,0,0),(1,0,0),(0,1,0),(-1,1,0),(-1,0,0)$, $(0,-1,0)$ and $(1,-1,0)$. In this case, the potential energy of the loop computed from (45) reduces to

$\varepsilon=\frac{b^{2}(47 \alpha+25 \beta-22 \gamma)}{8}-\frac{1}{(2 \pi)^{3}} \int_{B} \frac{1}{2} D_{k i}^{-1}(\boldsymbol{k}) \hat{f}_{k}^{\mathrm{E} *}(\boldsymbol{k}) \hat{f}_{i}^{\mathrm{E}}(\boldsymbol{k}) \mathrm{d}^{3} k-\sum_{\boldsymbol{l}} F_{i}^{\mathrm{E}}(\boldsymbol{l}) u_{i}^{\mathrm{S}}(\boldsymbol{l})$

and these are tabulated in table 5 for various materials. The externally applied stress required to stabilize the loop is also shown in table 5. It is seen that in this case the nucleation is not 
Table 5. Potential energy (eV), activation energy $(\mathrm{eV})$ and resolved shear stress for a hexagonal loop in FCC crystals.

\begin{tabular}{lrlll}
\hline Material & \multicolumn{1}{c}{$\varepsilon$} & \multicolumn{1}{l}{$\lambda_{\mathrm{S}}$} & $\varepsilon^{\mathrm{a}}$ & $\tau / \mu$ \\
\hline $\mathrm{Ag}$ & -7.037581 & 0.117911 & 0.128037 & 0.465264 \\
$\mathrm{Al}$ & -7.034164 & 0.125969 & 0.149213 & 0.445600 \\
$\mathrm{Au}$ & -13.993365 & 0.043868 & 0.029518 & 0.495855 \\
$\mathrm{Cu}$ & -5.880701 & 0.174545 & 0.275247 & 0.439127 \\
$\mathrm{Ni}$ & -8.140570 & 0.207642 & 0.600260 & 0.420614 \\
$\mathrm{~Pb}$ & -6.046817 & 0.054199 & 0.019922 & 0.508019 \\
$\mathrm{Th}$ & -9.821797 & 0.136834 & 0.253190 & 0.480566 \\
\hline
\end{tabular}
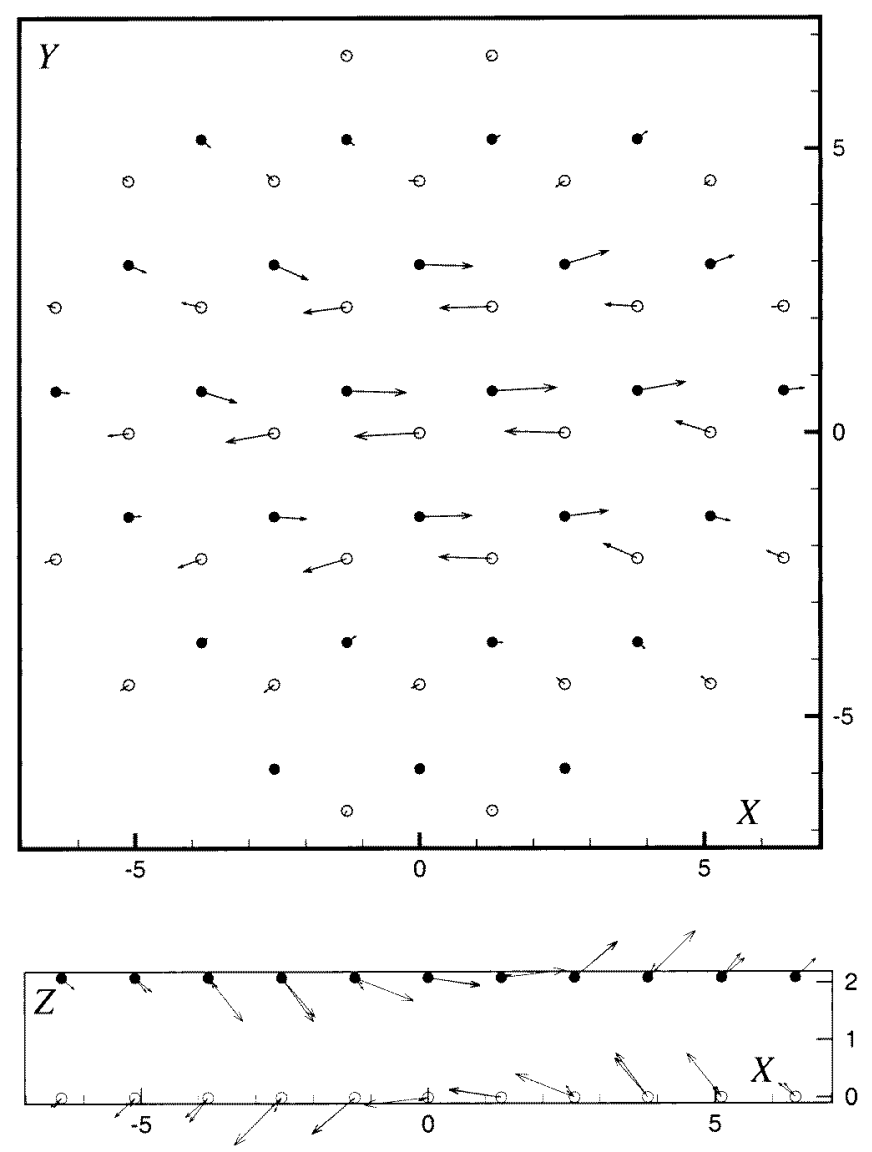

Figure 3. An arrow plot of displacements due to a hexagonal dislocation loop in $\mathrm{Cu}$. Open circles denote the atoms on the plane $l^{3}=0$ while full circles represent atoms on the plane $l^{3}=1$. The longest arrow in the top figure corresponds to $0.244 \AA$. The $\mathrm{X}$ axis corresponds to a $(011)$ crystal direction, Y corresponds to $(2 \overline{1} 1)$ and $\mathrm{Z}$ to (11) $)$. Length is in $\AA$. The displacement due to the homogeneous strain is not included.

spontaneous - an activation barrier needs to be overcome. An arrow plot of the displacements corresponding to the hexagonal loop for the case of $\mathrm{Cu}$ is given in figure 3 . 
As an illustration of the application of this theory to the growth of loops we now consider a loop where slip occurs on two adjacent atoms on the slip plane. Two distinct possibilities arise-case 1: a unit eigendistorsion at $(0,0,0)$ is superposed with a unit eigendistorsion at $(1,0,0)$; case 2 : a unit eigendistorsion at $(0,0,0)$ is superposed with a unit eigendistorsion at $(0,1,0)$. It is interesting to find out which of these cases is more easily activated. In case 1 , the energy expression (45) reduces to

$\varepsilon=\frac{b^{2}(11 \alpha+7 \beta-4 \gamma)}{8}-\frac{1}{(2 \pi)^{3}} \int_{B} \frac{1}{2} D_{k i}^{-1}(\boldsymbol{k}) \hat{f}_{k}^{\mathrm{E} *}(\boldsymbol{k}) \hat{f}_{i}^{\mathrm{E}}(\boldsymbol{k}) \mathrm{d}^{3} k-\sum_{l} F_{i}^{\mathrm{E}}(\boldsymbol{l}) u_{i}^{\mathrm{S}}(\boldsymbol{l})$

while in case 2 one obtains

$\varepsilon=\frac{3 b^{2}(2 \alpha+\beta-\gamma)}{4}-\frac{1}{(2 \pi)^{3}} \int_{B} \frac{1}{2} D_{k i}^{-1}(\boldsymbol{k}) \hat{f}_{k}^{\mathrm{E} *}(\boldsymbol{k}) \hat{f}_{i}^{\mathrm{E}}(\boldsymbol{k}) \mathrm{d}^{3} k-\sum_{l} F_{i}^{\mathrm{E}}(\boldsymbol{l}) u_{i}^{\mathrm{S}}(\boldsymbol{l})$.

We now calculate the activation energy for the growth of the single site loop to the double site loop in these two cases using expressions (48) and (49). The results are given in table 6 for case 1 and table 7 for case 2 . The externally applied stress in both cases is the same as that shown in table 4 . It is seen that in case 1 , the values of $\lambda_{\mathrm{s}}$ are all between 0 and 1 -an activation barrier has to be overcome-while in case 2 , the process of growth is spontaneous at the applied stress level.

Table 6. Potential energy (eV), activation energy $(\mathrm{eV})$ for the growth of a loop from a single site to a double site. Case 1: loop grows from $(0,0,0)$ to include $(1,0,0)$.

\begin{tabular}{llll}
\hline Material & $\varepsilon$ & $\lambda_{\mathrm{S}}$ & $\varepsilon^{\mathrm{a}}$ \\
\hline $\mathrm{Ag}$ & -1.867168 & 0.230146 & 0.057852 \\
$\mathrm{Al}$ & -2.239120 & 0.020177 & 0.000384 \\
$\mathrm{Au}$ & -3.528251 & 0.290593 & 0.194910 \\
$\mathrm{Cu}$ & -1.595584 & 0.247864 & 0.060628 \\
$\mathrm{Ni}$ & -2.473846 & 0.162937 & 0.035393 \\
$\mathrm{~Pb}$ & -1.446044 & 0.293866 & 0.082345 \\
$\mathrm{Th}$ & -2.363482 & 0.178146 & 0.040844 \\
\hline
\end{tabular}

Table 7. Potential energy $(\mathrm{eV})$, activation energy $(\mathrm{eV})$ for the growth of a loop from a single site to a double site. Case 2: loop grows from $(0,0,0)$ to include $(0,1,0)$.

\begin{tabular}{llll}
\hline Material & $\varepsilon$ & $\lambda_{\mathrm{S}}$ & $\varepsilon^{\mathrm{a}}$ \\
\hline $\mathrm{Ag}$ & -2.889462 & -0.237839 & 0.0 \\
$\mathrm{Al}$ & -2.662937 & -0.204518 & 0.0 \\
$\mathrm{Au}$ & -5.494946 & -0.135439 & 0.0 \\
$\mathrm{Cu}$ & -2.607976 & -0.265085 & 0.0 \\
$\mathrm{Ni}$ & -3.619259 & -0.266650 & 0.0 \\
$\mathrm{~Pb}$ & -2.370932 & -0.191113 & 0.0 \\
$\mathrm{Th}$ & -3.816496 & -0.386355 & 0.0 \\
\hline
\end{tabular}

\section{Summary and conclusions}

In this paper, we have extended harmonic lattice statics to allow for the emergence of lattice defects. This extension relies on Mura's theory of eigendistorsions. Application of the theory to FCC crystals has provided us with the following insights. Thermal activation of even the 
smallest of loops is unlikely in an unstressed crystal. Much larger stresses, of the order of $90 \%$ of the theoretical strength of the crystal, are required to stabilize the smallest loop and, if such stresses are exerted on the crystal, the nucleation is spontaneous. Stresses of such magnitude may be found near crack tips and second-phase particles. Larger loops such as an hexagonal loop enclosing seven atoms, may be nucleated at lower stresses of the order of $70 \%$ of the strength of the crystal. In this case, however, the nucleation is not spontaneous but involves an activation barrier ranging from 0.01 to $0.6 \mathrm{eV}$ in the metals that we have studied. We, therefore, conclude that thermally activated nucleation of dislocation loops is likely only in the vicinity of severe stress risers such as crack tips. Our analysis also suggests that, once nucleated, some loops may spontaneously grow along preferred directions in order to reduce the potential energy of the crystal.

In general, our analysis supports the theory put forward by Khantha et al (1994), in as much as it suggests that the thermally assisted nucleation of large numbers of small dislocations is indeed possible, with the proviso that such proliferation is only likely when the crystal is very severely stressed. However, since the theory of Khantha et al (1994) was primarily motivated by a desire to understand the BDT in crystals, the conditions of interest are those attained near the tip of a crack at impending growth, where the level of stress may indeed be high enough for thermally activated nucleation of small dislocation loops to operate. It must be noted that the present calculations are performed under conditions of an homogeneous stress background while the conditions near a crack tip or other stress risers are quite inhomogeneous. Also, the collective behaviour of dislocations is not addressed.

Although the theory developed in this paper has the virtue of being simple and analytically tractable, we hasten to emphasize its many approximations. While the use of eigendistorsions does supply the non-convexity required for the emergence of defects, it may not accurately represent the actual energy landscape of the crystal far from energy-wells. Another limitation is the assumption of nearest neighbour interactions between lattice sites. This assumption is strictly a matter of convenience and more general force-constant models may be considered, albeit with some additional labour.

In conclusion, we note that the problem of nascent dislocations in crystals does not in any way exhaust the catalogue of problems which may be addressed using the lattice theory of eigendistorsions. To give a concrete example, the nucleation of small misfit dislocation loops at the interface between a thin film and a substrate, such as observed in the experiments of Perovic and Houghton (1993), and other similar problems, should yield to methods similar to those employed in this paper.

\section{References}

Begbie G H 1947 Proc. R. Soc. A 188 189-208

Born M and Huang K 1954 Dynamical Theory of Crystal Lattices (Oxford: Oxford University Press)

Born M and Oppenheimer R 1927 Ann. Phys. Lpz 84 457-84

Gallego R and Ortiz M 1993 Modelling Simul. Mater. Sci. Eng. 1 417-36

Khantha M, Pope D P and Vitek V 1994 Phys. Rev. Lett. 73 684-7

Kosterlitz J M and Thouless D J 1973 J. Phys. C: Solid State Phys. 6 1181-203

Kroupa F 1966 Theory of Crystal Defects - Proc. Summer School (Hrazany, September 1964) ed B Gruber (New York: Academic)

Mura T 1987 Micromechanics of Defects in Solids (Boston, MA: Kluwer)

Nabarro F R N 1967 Theory of Crystal Dislocations (Oxford: Oxford University Press)

Oh D J and Johnson R A 1988 J. Mater. Res. 3 471-8

Perovic D D and Houghton D C 1993 Phys. Stat. Sol. a 138 425-30 